\title{
IS THERE ANY JUSTIFICATION FOR USING OZONETHERAPY IN HUMAN PATHOLOGIES CAUSED OR WORSENED BY FREE RADICALS?
}

\author{
"It is better to stir up a question without deciding it, than to decide it without stirring it up" \\ Joseph Joubert
}

The involvement of ROS in several human diseases is widely accepted, although it remains uncertain whether increased ROS formation is the primary cause or an epiphenomenon that simply contributes to progression of the disease (Halliwell et al., 1992, 2000; Ames et al., 1993; McCall and Frei, 1999; Fukagawa, 1999). An example of the former possibility can be found in neurodegenerative processes where a defect of antioxidants appears to be the main culprit, such as a defective SOD in amyotrophic lateral sclerosis and GSH in Parkinson's disease (Jenner, 1994; Bondy, 1995; Simonian and Coyle, 1996; Rowland and Shneider, 2001; Ferri et al., 2001). On the other hand, viral infections, such as hepatitis $\mathrm{C}$ virus (HCV) or human immunodeficiency virus (HIV), begin with viral contamination; after the infection is established, the virus changes the cell environment to its advantage and by various mechanisms induces a persistent oxidative stress due to an imbalance between antioxidants and pro-oxidants in favour of the latter (Larrea et al., 1998). The etiology of autoimmune diseases (lupus erythematosus, rheumatoid arthritis, multiple sclerosis, diabetes, Crohn's disease, etc.) remains undefined. However, it is likely due to a primary infection which, in a genetically predisposed individual, leads to a deranged immune response: an abnormal, cytotoxic $\mathrm{T}$ cell clone develops and, by recognizing an autoantigen on some cells, destroys them. Atherogenesis appears somewhat atypical but one of the primary events appears to be oxidation of Low Density Lipoproteins (LDL) which, via the scavenger receptor A (SRA), are internalized in endothelial cells as a first step in increasing the cholesterol content (Berliner and Heinecke, 1996; Steinberg, 1997). In any case, once the primary event is established, activation of phagocytes, of lipoxygenases, metal-ion release and deterioration of mitochondrial electron transport chains enhance the formation of $\mathrm{O}_{2}{ }^{\circ}, \mathrm{H}_{2} \mathrm{O}_{2}, \mathrm{OH}^{\bullet}, \mathrm{NO}^{\bullet}, \mathrm{ONOO}^{-}$and $\mathrm{HOCl}$, with tissue damage and cell death.

In rheumatoid arthritis patients, neutrophil granulocytes are attracted into the synovial fluid by chemotactic factors and contribute to joint injury. A similar situation seems to occur in adult respiratory distress syndrome (ARDS): by releasing ROS and serine proteinases, leukocytes inactivate $\alpha_{1}$-antiproteinase so that elastase breaks down pulmonary elastic fibres. Ischaemia may favour the release of $\mathrm{Fe}^{2+}$ into the anoxic area so that, during reperfusion, formation of $\mathrm{OH}^{\bullet}$ by the Fenton type chemistry can lead to irreversible tissue damage (Gutteridge and Halliwell, 1990). 
Oxidative stress has also been documented during strenuous physical competition ( $\mathrm{Ji}$, 1995; Sen, 1995), such as the marathon, and even in ageing, albeit in a milder but continuous way. Harman (1956) was the first to propose the free radical theory of ageing by assuming that ROS continually damage lipids, proteins and DNA. It has been well established that the common denominator in all these diseases is that, once started, oxidative stress goes on all the time until death (Halliwell et al., 1992; Ames et al., 1993; Yu, 1994, 1996; Meydani et al., 1995; Halliwell, 1996, 1999b, 2001; Beckman and Ames, 1998; Floyd, 1999; Fukagawa, 1999; Hamilton et al., 2001).

Why is it that living organisms that have been able to counteract the offensive action of oxygen during evolution can tolerate and then succumb to oxidative stress? It seems as if this chronic state induces a sort of anergy or a total inability to reverse the situation. There may be some spontaneous remissions or quieter phases, and administration of antioxidants (since they are not harmful) may ameliorate or delay, but not reverse, the oxidative stress. Dietary restrictions ( $\mathrm{Yu}$, 1996; Hamilton et al., 2001) may be helpful, but they are difficult to implement and can yield uncertain results in patients. While nobody can doubt the efficacy of the disinfectant properties of ozone, everyone knows that ozone can be toxic. Therefore, is it rational to propose ozonetherapy?

Dr. E. Payr (1871-1946) and Dr. E. Fisch (1899-1966) were the first to propose the medical use of ozone. Yet, in those days, orthodox medicine had little to offer and their pioneering idea could be justified. Today, the situation is radically different and the proposal of a medical therapy based on ozone seems to border on insanity. Nevertheless, a large body of medical evidence produced during the last decade, even though anecdotal and fragmentary, compels our attention. One of the aims of this book is to search for valid reasons to use ozone and to point out mistakes and false claims. Most important, however, is to demonstrate that the toxicity of ozone can be tamed and to evaluate whether ozonetherapy is truly efficacious.

The hypothesis that ozone can reverse chronic oxidative stress (Bocci, 1996a,d, 1988c; Leon et al., 1998; Barber et al., 1999) when we know (Chapters 5 and 9) that ozone is a master generator of ROS sounds paradoxical. What then are the ideas behind the proposal to verify or refute the validity of ozonetherapy?

1) The toxicity of ozone as it is used for $\mathrm{O}_{3}$-AHT can be well controlled and there is overwhelming evidence that is insignificant. The oxidative stress imposed on the blood is "calculated" (on the basis of ozone/blood concentrations) and is extremely "transitory". In fact, rather than talking of an acute oxidative stress, it would be preferable to introduce the concept of a "multivaried therapeutic response following small and repeated oxidative stresses" or simply of a therapeutic "shock".

2) The therapeutic "shock" is exogenous, i.e. it occurs ex vivo in a precisely controlled situation and, as such, must not be added to the chronic oxidative stress (COS) occurring inside the cell. It appears most unlikely that ROS generated during the therapeutic "shock" can reach and damage leukocytic DNA.

3) The amounts of LOPs produced ex vivo during $\mathrm{O}_{3}$-AHT (normally $225 \mathrm{ml}$ of blood $+225 \mathrm{ml}$ of $\mathrm{O}_{2}-\mathrm{O}_{3}$, with $\mathrm{O}_{3}$ concentrations in the range $20-80 \mu \mathrm{g} / \mathrm{ml}$; total 
doses ranging from 4.5 to $18 \mathrm{mg} \mathrm{O}_{3}$ ) are under control. The "therapeutic response" is generated by ROS and LOPs derived from plasma. Reinfusion occurs in 20-30 min and involves a 600 - to 700 -fold dilution because about $7 \mathrm{ml}$ of ozonized blood re-enter the circulation and are mixed with about $5 \mathrm{~L}$ of plasma every minute. The final LOP plasma levels depend on diffusion into about $10 \mathrm{~L}$ of extracellular fluid, as well as on receptor binding, catabolism and renal filtration. As a consequence and as we have observed, LOPs disappear rapidly from plasma in vivo. Therefore, the therapeutic "shock" has a unique pharmacokinetic characteristic: reinfusion of ozonized blood involves only a transient pulse of ROS that can exert a stimulus at variance with the constant ROS levels, as observed in patients with degenerative-inflammatory diseases.

4) Another critical difference between endogenous and therapeutic "shock" is that the intermittent schedule of $\mathrm{O}_{3}$-AHT appears to induce an upregulation of antioxidant enzymes which, in comparison to the administration of antioxidants, is one possible way of reversing chronic oxidative stress. Evidence for this is discussed in Chapter 22. The therapeutic "shock" may well induce peculiar oxidative stress proteins (OSP), such as haeme oxygenase (HSP32) or other chaperones, able to display therapeutic activities in vasculopathies, viral infections and cancer.

5) Great care must be taken in ensuring that patients undergoing $\mathrm{O}_{3}$-AHT have a plasmatic total antioxidant status (TAS; Miller et al., 1993) within the normal range (1.3-1.8 mM). Depletion of antioxidants can be suspected after anorexia, malnutrition, cachexia, chemotherapy, etc. Therefore, bearing in mind the axiom "primum non nocere" (first, do not harm), the basic TAS level must be checked. If it is below the normal value, standard pharmaceutical supplements must be administered daily for at least two weeks before starting ozonetherapy. In Chapter 12 , the evidence that plasma antioxidants constitute a strong defense against ozone will be provided.

It is possible that, in future, gene therapy or the use of stem cells may work miracles and make ozonetherapy truly obsolete. But when? 